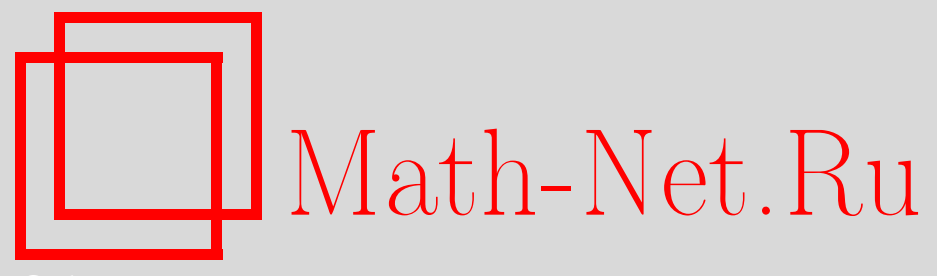

Ю. Л. Павлов, Предельные теоремы для объемов деревьев непомеченного графа случайного отображения, Дискрет. матем., 2004, том 16, выпуск 3, 63-75

DOI: https://doi.org/10.4213/dm163

Использование Общероссийского математического портала Math-Net.Ru подразумевает, что вы прочитали и согласны с пользовательским соглашением http://www . mathnet.ru/rus/agreement

Параметры загрузки:

IP : 54.197 .217 .227

26 апреля 2023 г., 17:55:07

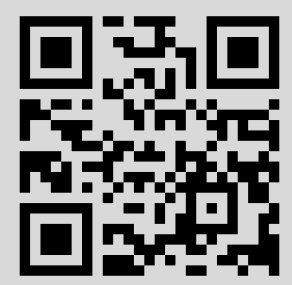




\title{
Предельные теоремы для объемов деревьев непомеченного графа случайного отображения
}

\author{
() 2004 г. . Л. Павлов
}

\begin{abstract}
Получены предельные распределения максимального объема дерева и числа деревьев заданного объема в непомеченном случайном лесе, состоящем из $N$ корневых деревьев и $n$ некорневых вершин, при $N, n \rightarrow \infty$ так, что $0<C_{1} \leqslant N / \sqrt{n} \leqslant C_{2}<\infty$. С помощью этих результатов для непомеченного графа случайного однозначного отображения множества $\{1,2, \ldots, n\}$ в себя при $n \rightarrow \infty$ доказаны теоремы о предельном поведении максимального объема дерева и числа деревьев объема $r$ в случае фиксированного $r$ и $r / n^{1 / 3} \geqslant C_{3}>0$.

Работа выполнена при поддержке гранта НШ 1758.2003.1 Президента Российской Федерации государственной поддержки ведущих научных школ Российской Федерации.
\end{abstract}

Случайные отображения являются одним из важнейших объектов исследования современной вероятностной комбинаторики. Наиболее полно результаты таких исследований представлены в книге В. Ф. Колчина “Случайные отображения” [1]. Хорошо известно, что однозначному отображению конечного множества в себя соответствует граф, вершинами которого являются элементы этого множества. Из каждой вершины такого графа выходит ровно одна дуга, ведущая в образ этой вершины. Заметим, что если элемент множества отображается в себя, то граф содержит петлю, а если два элемента отображаются друг в друга, то возникает ребро кратности два. Легко видеть, что каждая компонента связности графа отображения содержит один цикл, а циклические вершины служат корнями деревьев. Если убрать дуги, соединяющие циклические вершины, то оставшийся подграф представляет собой лес, состоящий из корневых деревьев, они называются деревьями отображения. Под объемом дерева будем понимать число вершин, содержащихся в этом дереве.

При решения перечислительных задач комбинаторики с помощью вероятностных методов обычно на множестве всех рассматриваемых объектов задают равномерное распределение вероятностей. В таком случае каждое однозначное отображение множества объема $n$ в себя появляется с вероятностью $n^{-n}$, а различные числовые характеристики отображения можно считать случайными величинами. Важными характеристиками являются максимальный объем дерева и число деревьев заданного объема. Слабая сходимость к предельным законам распределений таких характеристик впервые была доказана в статье В. Е. Степанова [2]. В [3, 4] удалось в некоторых случаях получить соответствующие локальные предельные теоремы, при этом использовались результаты о случайных лесах. В этих работах рассмотрено множество лесов, состоящих из $N$ корневых деревьев и содержащих $n$ некорневых вершин, все деревья и все вершины считались различными 
(помеченными). На множестве таких лесов было введено равномерное распределение вероятностей, и были доказаны предельные теоремы для максимального объема дерева и числа деревьев заданного объема. Эти результаты были использованы для изучения предельного поведения объемов деревьев случайного отображения путем усреднения распределений рассматриваемых характеристик лесов по известному [5] распределению числа циклических вершин случайного отображения.

Главным методом получения результатов в $[3,4]$ была обобщенная схема размещения частиц по ячейкам, введенная и исследованная В. Ф. Колчиным [1]. Такая схема задается двумя наборами целочисленных неотрицательных случайных величин $\left(\eta_{1}, \ldots, \eta_{N}\right)$ и $\left(\xi_{1}, \ldots, \xi_{N}\right)$, связанными соотношением

$$
\mathbf{P}\left\{\eta_{1}=k_{1}, \ldots, \eta_{N}=k_{N} t\right\}=\mathbf{P}\left\{\xi_{1}=k_{1}, \ldots, \xi_{N}=k_{N} \mid \xi_{1}+\ldots+\xi_{N}=n\right\},
$$

при этом $\eta_{1}+\ldots+\eta_{N}=n$, a $\xi_{1}, \ldots, \xi_{N}$ независимы.

Пусть $\mu_{r}$ означает число случайных величин $\eta_{1}, \ldots, \eta_{N}$, принявших значение $r$, a $\eta_{(N)}=\max \left(\eta_{1}, \ldots, \eta_{N}\right)$. Введем вспомогательные случайные величины $\xi_{1}^{(r)}, \ldots, \xi_{N}^{(r)}$, $\xi_{1}(r), \ldots, \xi_{N}(r)$ такие, что при $k=0,1,2, \ldots$

$$
\begin{aligned}
\mathbf{P}\left\{\xi_{1}^{(r)}=k\right\} & =\mathbf{P}\left\{\xi_{1}=k \mid \xi_{1} \neq r\right\}, \\
\mathbf{P}\left\{\xi_{1}(r)=k\right\} & =\mathbf{P}\left\{\xi_{1}=k \mid \xi_{1} \leqslant r\right\} .
\end{aligned}
$$

Положим также

$$
\begin{aligned}
p_{k} & =\mathbf{P}\left\{\xi_{1}=k\right\}, \\
\zeta_{N} & =\xi_{1}+\ldots+\xi_{N}, \\
\zeta_{N}^{(r)} & =\xi_{1}^{(r)}+\ldots+\xi_{N}^{(r)}, \\
\zeta_{N}(r) & =\xi_{1}(r)+\ldots+\xi_{N}(r), \\
P_{r} & =\mathbf{P}\left\{\xi_{1}>r t\right\} .
\end{aligned}
$$

В [1] показано, что в условиях обобщенной схемы размещения имеют место следующие утверждения.

Лемма 1. Справедливо равенство

$$
\mathbf{P}\left\{\mu_{r}=k\right\}=\left(\begin{array}{l}
N \\
k
\end{array}\right) p_{r}^{k}\left(1-p_{r}\right)^{N-k} \frac{\mathbf{P}\left\{\zeta_{N-k}^{(r)}=n-k r\right\}}{\mathbf{P}\left\{\zeta_{N}=n\right\}} .
$$

Лемма 2. Справедливо равенство

$$
\mathbf{P}\left\{\eta_{(N)} \leqslant r\right\}=\left(1-P_{r}\right)^{N} \frac{\mathbf{P}\left\{\zeta_{N}(r)=n\right\}}{\mathbf{P}\left\{\zeta_{N}=n\right\}} .
$$

В $[3,4]$ показано, что если $\eta_{1}, \ldots, \eta_{N}$ означают объемы деревьев случайного леса, а $\xi_{1}, \ldots, \xi_{N}$ имеют распределение Бореля-Таннера, то выполнено равенство (1) и леммы 1 и 2 можно применить для изучения предельного поведения числа деревьев заданного объема и максимального объема дерева в случайном лесе.

В ряде публикаций (см., например, $[6,7])$ рассматривалось множество $G$, состоящее из всех различных графов отображений с непомеченными вершинами. Это значит, что в $G$ графы отображений, отличающиеся только нумерацией вершин, отождествляются. 
Хорошо известно [7], что задачи перечисления непомеченных комбинаторных объектов решаются гораздо труднее, чем аналогичные задачи для помеченных объектов. Если, например, для числа помеченных деревьев и лесов и соответствующих производящих функций имеются простые формулы, то в случае непомеченных графов ситуация иная. Здесь можно отметить классическую работу [8], в которой рассматривалась задача перечисления непомеченных деревьев. Введем производящую функцию

$$
T(x)=\sum_{n=1}^{\infty} T_{n} x^{n},
$$

где $T_{n}$ - число корневых непомеченных деревьев объема $n$. В [8] рассмотрены свойства этой функции и установлено, что ряд (4) имеет радиус сходимости, равный $R=0,3383219 \ldots$ Кроме того, там показано, что

$$
T(R)=1
$$

а функция $T(x)$ может быть представлена в виде

$$
T(x)=1-b(R-x)^{1 / 2}+b_{1}(R-x)+b_{2}(R-x)^{3 / 2}+\ldots,
$$

где коэффициенты $b, b_{1}, b_{2}, \ldots$ определены в [8], в частности, $b=2,681127 \ldots$ Кроме того, при $n \rightarrow \infty$ для $T_{n}$ в [8] найдено асимптотическое представление

$$
T_{n}=\beta\left(R^{n} n^{3 / 2}\right)^{-1}+O\left(\left(R^{n} n^{5 / 2}\right)^{-1}\right),
$$

где $\beta=0,4399237 \ldots$

В [9] на множестве $G$ было введено равномерное распределение и получены предельные теоремы для некоторых характеристик непомеченных графов случайных отображений. Оказалось, что предельные распределения числа компонент непомеченных и помеченных графов отображений совпадают, а предельные распределения числа циклических вершин отличаются только значением параметра. Обозначим через $\lambda$ число циклических вершин непомеченного графа отображения с $n$ вершинами.

Лемма 3 ([9]). Если $n \rightarrow \infty u$ u $=l / \sqrt{n}$, где $l$ - положительное челое число, то

$$
\mathbf{P}\{\lambda / \sqrt{n}=u\}=\left(b^{2} R /(2 \sqrt{n})\right) u \exp \left\{-b^{2} R u^{2} / 4\right\}(1+o(1))
$$

для всех $и=o(\sqrt{n} / \ln n)$.

В настоящей работе при $n \rightarrow \infty$ получены предельные распределения случайной величины $\eta(G)$, равной максимальному объему дерева (теорема 4), и величин $\mu_{r}(G)$, равных числу деревьев объема $r$ в графе из $G$ для фиксированных $r$ и $r \rightarrow \infty$ так, что $r / n^{1 / 3} \geqslant C_{1}>0$ (теоремы 5-7). Для доказательства этих теорем рассматривается множество $F$ лесов, состоящих из $N$ корневых деревьев, упорядоченных одним из $N$ ! возможных способов, и $n$ некорневых непомеченных вершин. На этом множестве вводится равномерное распределение вероятностей. Обозначим через $\eta(N, n)$ и $\mu_{r}(N, n)$, соответственно, максимальный объем дерева и число деревьев объема $r$ в лесе из $F$. Для этих случайных величин получены предельные распределения при $N, \boldsymbol{n} \rightarrow \infty$ так, что $0<C_{1} \leqslant N / \sqrt{n} \leqslant C_{2}<\infty$ (теоремы 1-3). Это ограничение связано с тем, что, в соответствии с леммой 3 , число циклических вершин в графе из $G$ имеет порядок $\sqrt{n}$. Остальные зоны изменения параметров $N$ и $n$ будут рассмотрены в другой работе автора. 
Для доказательства теорем 1-3 была использована обобщенная схема размещения. Пусть случайные величины $\eta_{1}, \ldots, \eta_{N}$ означают объемы деревьев леса из $F$, имеющих номера $1, \ldots, N$, соответственно. Пусть также $\xi_{1}, \ldots, \xi_{N}$ имеют распределение

$$
p_{k}=\mathbf{P}\left\{\xi_{1}=k\right\}=T_{k} R^{k}, \quad k=1,2, \ldots,
$$

здесь мы учли (4) и (5). Нетрудно видеть, что заданные таким образом случайные величины удовлетворяют (1). Заметим, что изучение предельного поведения объемов деревьев непомеченного случайного леса является новым примером использования обобщенной схемы размещения. Справедливы следующие утверждения.

Теорема 1. Пусть $N, n \rightarrow \infty$ так, что $2 n /\left(b^{2} R N^{2}\right) \rightarrow \gamma$, где $\gamma$ - положсительная постоянная. Тогда для любого фиксированного $z>0$

$$
\mathbf{P}\left\{\frac{\eta(N, n)}{n} \leqslant z\right\} \rightarrow \gamma^{3 / 2} \exp \left\{\frac{1}{2 \gamma}\right\} \sum_{s=0}^{\infty} \frac{(-1)^{s}}{s !} I_{s}(z \gamma, \gamma)
$$

2de

$$
\begin{aligned}
I_{0}(u, v) & =\left(v^{3} \exp \{1 / v\}\right)^{-1 / 2} \\
I_{s}(u, v) & =\int_{X_{s}(u, v)} \frac{\exp \left\{-1 /\left(\left(v-x_{1}-\ldots-x_{s}\right)\right)\right\}}{(2 \pi)^{s / 2}\left(x_{1} \ldots x_{s}\left(v-x_{1}-\ldots-x_{s}\right)\right)^{3 / 2}} d x_{1} \ldots d x_{s}, \\
X_{s}(u, v) & =\left\{x_{i} \geqslant u, i=1, \ldots, s, x_{1}+\ldots+x_{s} \leqslant v\right\}, \quad s=1,2, \ldots
\end{aligned}
$$

Теорема 2. Пусть $N, n \rightarrow \infty$ так, что $0<C_{1} \leqslant N / \sqrt{n} \leqslant C_{2}<\infty, r=o\left(n^{1 / 3}\right)$, $u_{r}=\left(k-N p_{r}\right) / \sqrt{N p_{r}\left(1-N p_{r}\right)}$. Тогда для чельх неотричательных $k$ равномерно относительно $\left(k-N p_{r}\right) /\left(N p_{r}\left(1-p_{r}\right)\right)^{7 / 12}$ в любом фиксированном конечном интервале

$$
\mathbf{P}\left\{\mu_{r}(N, n)=k\right\}=\frac{1}{\sqrt{2 \pi N p_{r}\left(1-p_{r}\right)}} e^{-u_{r}^{2} / 2}(1+o(1)) .
$$

Теорема 3. Пусть $N, n, r \rightarrow \infty$ так, что $0<C_{1} \leqslant N / \sqrt{n} \leqslant C_{2}<\infty$. Тогда для чельц неотричательных $k$ равномерно относительно $\left(k-N p_{r}\right) / \sqrt{N p_{r}}$ в любом фиксированном конечном интервале

$$
\mathbf{P}\left\{\mu_{r}(N, n)=k\right\}=\frac{\left(N p_{r}\right)^{k}}{k !} e^{-N p_{r}}(1+o(1)) .
$$

Теорема 4. При $n \rightarrow \infty$ для любого фиксированного $z>0$

$$
\mathbf{P}\{\eta(G) / n \leqslant z\} \rightarrow 1+\sum_{s=1}^{\infty} \frac{(-1)^{s} \Gamma(s / 2+1)}{s ! \pi^{s / 2}} \int_{X} \frac{\left(1-x_{1}-\ldots-x_{s}\right)^{(s-1) / 2}}{\left(x_{1} \ldots x_{s}\right)^{3 / 2}} d x_{1} \ldots d x_{s},
$$

где $\Gamma(x)$ - гамма-функиия, $X=\left\{x_{i} \geqslant z, i=1, \ldots, s, \sum_{i} x_{i} \leqslant 1\right\}$.

Теорема 5. Пусть $n \rightarrow \infty, r$ фиксировано, $z=k b \sqrt{R} /\left(p_{r} \sqrt{2 n}\right)$, где $k-$ иелое положительное число. Тогда

$$
\left(p_{r} \sqrt{2 n} /(b \sqrt{R})\right) \mathbf{P}\left\{b \sqrt{R} \mu_{r}(G) /\left(p_{r} \sqrt{2 n}\right)=z\right\}=z e^{-z^{2} / 2}+o(1)
$$

равномерно относительно $z$ в любом фиксированном конечном интервале вида $0<z_{0} \leqslant z \leqslant z_{1}<\infty$. 
Теорема 6. Если $n \rightarrow \infty, r=\left(2 \beta^{2} n /\left(\gamma^{2} b^{2} R\right)\right)^{1 / 3}+O(1), 0<\gamma<\infty$, то для любого фиксированного $k=0,1,2, \ldots$

$$
\mathbf{P}\left\{\mu_{r}(G)=k\right\} \rightarrow \int_{0}^{\infty}(\gamma y)^{k}\left(k ! e^{\gamma y}\right)^{-1} y e^{-y^{2} / 2} d y .
$$

Теорема 7. Если $n \rightarrow \infty, r / n^{1 / 3} \rightarrow \infty$, то $\mathbf{P}\left\{\mu_{r}(G)=0\right\} \rightarrow 1$.

Замечание 1. Сравнивая утверждения теорем 1-7 с аналогичными результатами для лесов и графов отображений с помеченными вершинами (см. $[1,3,4])$, легко видеть, что во всех случая х имеют место сходные предельные распределения, при этом в условиях теорем 1-3, 5 и 6 они отличаются только значениями параметров, а в условиях теорем 4 и 7 предельные распределения совпадают.

Замечание 2. Для любого $z$ в правой части утверждения теоремы 4 находится конечная сумма, в частности, при $1 / 2<z \leqslant 1$,

$$
\mathbf{P}\{\eta(G) / n \leqslant z\} \rightarrow 2-1 / \sqrt{z} .
$$

Ниже приводятся вспомогательные утверждения (леммы 4-7), с помощью которых далее будут доказаны теоремы 1-7. Символы $C_{1}, C_{2}, \ldots$, встречающиеся в доказательствах теорем и лемм, означают некоторые положительные постоянные.

Лемма 4. Пусть $N \rightarrow \infty$. Тогда для натуральных $h$ равномерно относительно $v=2 h /\left(b^{2} R N^{2}\right)$ в любом фиксированном конечном интервале вида $0<v_{0} \leqslant v \leqslant v_{1}$

$$
b^{2} R N^{2} 2^{-1} \mathbf{P}\left\{2 \zeta_{N} /\left(b^{2} R N^{2}\right)=v\right\}=\left(2 \pi v^{3} \exp \{1 / v\}\right)^{-1 / 2}(1+o(1)) .
$$

Доказательство. Обозначим через $\varphi(t)$ характеристическую функцию случайной величины $\xi_{1}$. Из (4), (6), (8) следует, что

$$
\varphi(t)=T\left(R e^{i t}\right)=1-b \sqrt{R}\left(1-e^{i t}\right)^{1 / 2}+b_{1} R\left(1-e^{i t}\right)+\ldots
$$

Отсюда нетрудно получить, что при любом фиксированном $t$

$$
\varphi^{N}\left(2 t /\left(b^{2} R N^{2}\right)\right)=\exp \{-\sqrt{-2 i t}\}(1+o(1)) .
$$

Последнее выражение, как хорошо известно (см., например, [1]), является характеристической функией устойчивого закона с показателем $\alpha=1 / 2$, имеющего плотность $\left(2 \pi x^{3} \exp \{1 / x\}\right)^{-1 / 2}$. Отсюда и из локальной предельной теоремы о сходимости распределений сумм независимых слагаемых (теорема 4.2.1 из [10]) следует утверждение леммы 4.

Лемма 5. Пусть $N, n \rightarrow \infty$ так, что $2 n /\left(b^{2} R N^{2}\right) \rightarrow \gamma, r=n z+O(1)$, где $\gamma, z-$ некоторые положительные постоянные. Тогда

$$
N P_{r} \rightarrow(2 \pi)^{-1 / 2} \int_{\gamma z}^{\infty} y^{-3 / 2} d y .
$$

Доказательство. Из (7) и (8) следует, что

$$
N P_{r}=N \beta(1+o(1)) \sum_{s=1}^{\infty}(r+s)^{-3 / 2} .
$$


Полагая $y=2(r+s) /\left(b^{2} R N^{2}\right)$ и заменяя суммирование интегрированием, получаем, что

$$
N P_{r}=\beta \sqrt{2 /\left(b^{2} R\right)} \int_{\gamma z}^{\infty} y^{-3 / 2} d y+o(1)
$$

Утверждение леммы 5 следует отсюда и из известного [8] равенства

$$
\beta=b \sqrt{R} /(2 \sqrt{\pi}) .
$$

Лемма 6. Пусть $N, n \rightarrow \infty$ так, что $2 n /\left(b^{2} R N^{2}\right) \rightarrow \gamma$, где $\gamma$ - некоторая положительная постоянная. Пусть $v=2 h /\left(b^{2} R N^{2}\right)$, где $h-$ натуральное число. Если $r=n z+O(1)$, где $z$ - фиксированное положстельное число, то равномерно относительно $v$ в любом фиксированном конечном интервале вида $0<v_{0} \leqslant v \leqslant v_{1}$

$$
\frac{b^{2} R N^{2}}{2} \mathbf{P}\left\{\frac{2 \zeta_{N}(r)}{b^{2} R N^{2}}=v\right\} \rightarrow \frac{1}{\sqrt{2 \pi}} e^{E(0, z)} \sum_{s=0}^{\infty} \frac{(-1)^{s}}{s !} I_{s}(v z, v)
$$

гde

$$
E(u, z)=(2 \pi)^{-1 / 2} \int_{\gamma z}^{\infty} y^{-3 / 2} e^{i u y} d y,
$$

a интегралы $I_{s}(u, v)$ определены в формулировке теоремы 1.

Доказательство. Обозначим через $\psi_{r}(t)$ характеристическую функщию случайной величины $2 \zeta_{N}(r) /\left(b^{2} R N^{2}\right)$. Тогда, используя (3) и (10), находим, что

$$
\begin{aligned}
\psi_{r}(t)=\left(1-P_{r}\right)^{-N} \exp \{ & -\sqrt{-2 i t}\} \\
& \times\left(1-(1+o(1)) \sum_{s=1}^{\infty} p_{r+s} \exp \left\{2 i t(r+s) /\left(b^{2} R N^{2}\right)\right\}\right)^{N} .
\end{aligned}
$$

Из (8), (12), (13) получаем, аналогично (11), что

$$
\sum_{s=1}^{\infty} p_{r+s} \exp \left\{2 i t(r+s) /\left(b^{2} R N^{2}\right)\right\}=E(t, z) / N(1+o(1)), \quad P_{r}=E(0, z) / N(1+o(1)) .
$$

Отсюда и из (14) следует, что

$$
\psi_{r}(t)=\exp \{-\sqrt{-2 i t}-E(t, z)+E(0, z)\}+o(1) .
$$

Поскольку $E(t, z)$ является преобразованием Фурье функции

$$
f(x)= \begin{cases}\left(\sqrt{2 \pi x^{3}}\right)^{-1} & \text { при } x \geqslant \gamma z \\ 0 & \text { при } x<\gamma z\end{cases}
$$

разлагая $\exp \{-E(t, z)\}$ в ряд по степеням $E(t, z)$ и учитывая явный вид плотности распределения устойчивого закона с показателем $\alpha=1 / 2$ (см. лемму 4), находим, что $\psi_{r}(t)$ сходится к характеристической функции распределения с плотностью

$$
g(v)=\frac{1}{\sqrt{2 \pi}} e^{E(0, z)} \sum_{s=0}^{\infty} \frac{(-1)^{s}}{s !} I_{s}(\gamma z, v) .
$$


В отличие от доказательства леммы 4 мы не можем далее непосредственно использовать локальную предельную теорему, поскольку параметр $r$ зависит от $n$ и, следовательно, случайные величины $\xi_{1}(r), \ldots, \xi_{N}(r)$ образуют схему серий. Утверждение леммы 6 нетрудно получить, дословно повторяя доказательство леммы 3.3.1 из [1].

Лемма 7. Пусть $N \rightarrow \infty, S=N\left(1-p_{r}+o\left(p_{r}\right)\right), v=2 h /\left(b^{2} R N^{2}\right)$, где $h-$ натуральное число. Тогда равномерно относительно $v$ в любом фиксированном конечном интервале вида $0<v_{0} \leqslant v \leqslant v_{1}$

$$
\frac{b^{2} R N^{2}}{2} \mathbf{P}\left\{\frac{2 \zeta S_{S}^{(r)}}{b^{2} R N^{2}}=v\right\}=\frac{1}{\sqrt{2 \pi v^{3}}} e^{-1 /(2 v)}(1+o(1))
$$

Доказательство. Обозначим через $\psi^{(r)}(t)$ характеристическую функщию случайной величины $2 \zeta_{S}^{(r)} /\left(b^{2} R N^{2}\right)$. Тогда, согласно (2) и (9),

$$
\psi^{(r)}(t)=\varphi^{S}\left(2 t /\left(b^{2} R N^{2}\right)\right)\left(\frac{1-\varphi^{-1}\left(2 t /\left(b^{2} R N^{2}\right)\right) p_{r} \exp \left\{-2 i t r /\left(b^{2} R N^{2}\right)\right\}}{1-p_{r}}\right)^{S}
$$

Из (10) нетрудно получить, что при любом фиксированном $t$

$$
\begin{aligned}
\varphi^{S}\left(2 t /\left(b^{2} R N^{2}\right)\right) & =\exp \left\{-\left(1-p_{r}\right) \sqrt{-2 i t}\right\}(1+o(1)), \\
\left(\frac{1-\varphi^{-1}\left(2 t /\left(b^{2} R N^{2}\right)\right) p_{r} \exp \left\{-2 i t r /\left(b^{2} R N^{2}\right)\right\}}{1-p_{r}}\right)^{S} & =\exp \left\{-p_{r} \sqrt{-2 i t}\right\}(1+o(1))
\end{aligned}
$$

поэтому из (17) находим, что

$$
\psi^{(r)}(t) \rightarrow \exp \{-\sqrt{-2 i t}\}
$$

Отсюда и из теоремы 4.2.1 [10] получаем, что при фиксированных $r$ имеет место указанная в утверждении леммы локальная сходимость.

Если $r \rightarrow \infty$, то случайные величины $\xi_{1}^{(r)}, \xi_{2}^{(r)}, \ldots$ образуют схему серий. Докажем локальную сходимость в этом случае.

Используя формулы обращения, получаем, что

$$
\begin{aligned}
\mathbf{P}\left\{\frac{2 \zeta_{S}^{(r)}}{b^{2} R N^{2}}=v\right\} & =\frac{1}{\pi b^{2} R N^{2}} \int_{-\pi b^{2} R N^{2} / 2}^{\pi b^{2} R N^{2} / 2} e^{-i t v} \psi^{(r)}(t) d t, \\
\left(2 \pi v^{3} \exp \{1 / v\}\right)^{-1 / 2} & =(2 \pi)^{-1} \int_{-\infty}^{\infty} \exp \{-i t v-\sqrt{-2 i t} t\} d t .
\end{aligned}
$$

Отсюда следует, что разность

$$
R_{S}=\pi b^{2} R N^{2} \mathbf{P}\left\{2 \zeta_{S}^{(r)} /\left(b^{2} R N^{2}\right)=v\right\}-\sqrt{2 \pi}\left(v^{3} \exp \{1 / v\}\right)^{-1 / 2}
$$


можно представить в виде суммы $R_{S}=I_{1}+I_{2}+I_{3}+I_{4}+I_{5}$, где

$$
\begin{aligned}
& I_{1}=\int_{-A}^{A} e^{-i t v}\left(\psi^{(r)}(t)-\exp \{-\sqrt{-2 i t}\}\right) d t, \\
& I_{2}=\int_{A<|t| \leqslant b^{4} R^{2} N^{2} /\left(2 r^{2}\right)} e^{-i t v} \psi^{(r)}(t) d t, \\
& I_{3}=\int_{b^{4} R^{2} N^{2} /\left(2 r^{2}\right)<|t| \leqslant \varepsilon N^{2}} e^{-i t v} \psi^{(r)}(t) d t, \\
& I_{4}=\int_{\varepsilon N^{2}<|t| \leqslant \pi b^{2} R N^{2} / 2} e^{-i t v} \psi^{(r)}(t) d t, \\
& I_{5}=-\int_{A<|t|} \exp \{-i t v-\sqrt{-2 i t}\} d t,
\end{aligned}
$$

положительные постоянные $A$ и $\varepsilon$ будут выбраны позднее.

Заметим, что если $b^{4} R^{2} N^{2} /\left(2 r^{2}\right)<A$, то область интегрирования $I_{2}$ пуста, в этом случае в качестве нижнего предела интегрирования $I_{3}$ возьмем $A$.

Для того чтобы завершить доказательство леммы, достаточно показать, что каждый из интегралов $I_{1}-I_{5}$ можно сделать сколь угодно малым при достаточно больших $N, n, A$ и достаточно малом $\varepsilon$.

Из (18) следует, что $I_{1} \rightarrow 0$ при любом фиксированном $A$. Поскольку

$$
\sqrt{-2 i t}=\sqrt{|t|}(1-i t /|t|)
$$

получаем, что величину $\left|I_{5}\right|$ можно сделать сколь угодно малой выбором достаточно большого $A$.

Оценим $I_{4}$. При $\varepsilon<|t| \leqslant \pi$, как известно, для некоторого $C_{1}$ справедливо неравенство $|\varphi(t)| \leqslant e^{-C_{1}}$. Отсюда следует, что при достаточно больших $r$

$$
\left|\mathbf{E} e^{i t \xi_{1}^{(r)}}\right| \leqslant \frac{|\varphi(t)|+p_{r}}{1-p_{r}} \leqslant e^{-C_{1} / 2},
$$

поскольку $p_{r} \rightarrow 0$ при $r \rightarrow \infty$. Поэтому

$$
\left|I_{4}\right| \leqslant C_{2} N^{2} e^{-C_{1} N / 2} \rightarrow 0 .
$$

Для оценки интеграла $I_{2}$ покажем, что если

$$
2|t| r^{2} /\left(b^{4} R^{2}\right) \leqslant 1,
$$

To

$$
\left|\mathbf{E} \exp \left\{\frac{2 i t \xi_{1}^{(r)}}{b^{2} R}\right\}\right|=\left|\frac{\varphi\left(2 t /\left(b^{2} R\right)\right)-p_{r} \exp \left\{2 i t r /\left(b^{2} R\right)\right\}}{1-p_{r}}\right| \leqslant\left|\varphi\left(2 t /\left(b^{2} R\right)\right)\right|^{1 / 2} .
$$

Из (6), (19) следует, что при $t \rightarrow 0$

$$
\varphi\left(2 t /\left(b^{2} R\right)\right)=\exp \{-\sqrt{|t|}(1-i t /|t|)+o(\sqrt{|t|})\} .
$$

Отсюда получаем, что

$$
\varphi\left(2 t /\left(b^{2} R\right)\right)=\rho(\cos \omega+i \sin \omega),
$$


где

$$
\rho=\exp \{-\sqrt{|t|}+o(\sqrt{|t|})\}, \quad \omega=t / \sqrt{|t|}+o(\sqrt{|t|})
$$

- действительные числа. Легко видеть, учитывая (20), что при достаточно малых $t$ справедливы неравенства

$$
\cos \omega \geqslant 1-\omega^{2} / 2 \geqslant 1-|t|, \quad \cos \left(2 \operatorname{tr} /\left(b^{2} R\right)\right) \geqslant 1-|t|,
$$

поэтому

$$
\cos \omega \cos \left(2 t r /\left(b^{2} R\right)\right) \geqslant 1-2|t| .
$$

При достаточно малых $t$, как легко видеть, $\rho \leqslant \exp \{-\sqrt{|t|} / 2\}$ и

$$
\cos \omega \cos \left(2 \operatorname{tr} /\left(b^{2} R\right)\right) \geqslant \exp \{-\sqrt{|t|} / 2\} \geqslant \rho .
$$

С помощью (20) нетрудно проверить, что при достаточно малых $t$ справедливо неравенстBO

$$
\sin \omega \sin \left(2 t r /\left(b^{2} R\right)\right) \geqslant 0,
$$

поэтому из (23) следует, что

$$
\cos \omega \cos \left(2 t r /\left(b^{2} R\right)\right)+\sin \omega \sin \left(2 t r /\left(b^{2} R\right)\right) \geqslant \rho .
$$

Отсюда и из (22) находим, что

$$
\left|\varphi\left(\frac{2 t}{b^{2} R}\right)-p_{r} \exp \left\{\frac{2 i t r}{b^{2} R}\right\}\right|^{2} \leqslant \rho^{2}+p_{r}^{2}-2 \rho^{2} p_{r} .
$$

Поскольку $p_{r} \rightarrow 0$ при $r \rightarrow \infty$ и величина $\rho$ может быть сделана сколь угодно близкой к единице, получаем, что $\rho^{2}+p_{r}^{2}-2 \rho^{2} p_{r}-\left(1-p_{r}\right)^{2} \rho \leqslant 0$. Отсюда и из (22), (24) следует (21).

Из (21), (9) и (10) вытекает, что в области интегрирования $I_{2}$ справедлива оценка

$$
\left|\psi^{(r)}\left(2 t /\left(b^{2} R N^{2}\right)\right)\right| \leqslant \exp \left\{-C_{3} \sqrt{|t|}\right\},
$$

поэтому

$$
\left|I_{2}\right| \leqslant \int_{A}^{\infty} \exp \left\{-C_{3} \sqrt{|t|}\right\} d t
$$

следовательно, $I_{2}$ можно сделать сколь угодно малым выбором $A$.

Ясно, что

$$
\mathbf{E} e^{i t \xi_{1}^{(r)}}=\varphi(t)\left(1+p_{r}\left(1-p_{r}\right)^{-1}\left(1-\varphi^{-1}(t) e^{i t r}\right)\right) .
$$

Отсюда и из (6)-(8), (19) получаем, что при достаточно больших $N$ и достаточно малом $\varepsilon$

$$
\left|\psi^{(r)}\left(\frac{2 t}{b^{2} R N^{2}}\right)\right| \leqslant \exp \left\{S\left(-\frac{C_{4} \sqrt{|t|}}{N}+\frac{C_{5}}{r^{3 / 2}}\right)\right\} .
$$

Отсюда и из условия $|t|>b^{4} R^{2} N^{2} /\left(2 r^{2}\right)$ находим, что в области интегрирования $I_{3}$ справедливы оценки, аналогичные (25) и (26), что и завершает доказательство леммы. 
Теперь мы можем доказать теоремы 1-7.

Теорема 1 очевидным образом следует из лемм 2, 4-6.

Если выполнены условия теоремы 2, то из лемм 4 и 7 получаем, что

$$
\mathbf{P}\left\{\zeta_{N-k}^{(r)}=n-k r\right\} / \mathbf{P}\left\{\zeta_{N}=n\right\} \rightarrow 1
$$

Из (7) и (8) следует, что при $r=o\left(n^{1 / 3}\right)$ имеет место соотношение $N p_{r}\left(1-p_{r}\right) \rightarrow \infty$. Хорошо известно (см., например, [1]), что при этом условии можно использовать нормальное приближение биномиальных вероятностей, справедливое равномерно относительно $\left(k-N p_{r}\right) /\left(N p_{r}\left(1-p_{r}\right)\right)^{7 / 12}$ в любом фиксированном конечном интервале. Отсюда, из леммы 1 и (27) получаем утверждение теоремы 2.

Ясно, что $p_{r} \rightarrow 0$ при $r \rightarrow \infty$, поэтому, используя леммы $1,4,7$ и пуассоновское приближение биномиальных вероятностей, приходим к утверждению теоремы 3.

Пусть выполнены условия теоремы 4. Нетрудно видеть, что

$$
\mathbf{P}\{\eta(G) / n \leqslant z\}=\sum_{N=1}^{n} \mathbf{P}\{\lambda=N\} \mathbf{P}\{\eta(N, n-N) / n \leqslant z\} .
$$

Отсюда и из леммы 3 получаем, что

$$
\mathbf{P}\{\eta(G) / n \leqslant z\}=\frac{b^{2} R}{2} \sum_{\varepsilon \leqslant N / \sqrt{n} \leqslant A} \frac{N}{n} \exp \left\{\frac{b^{2} R N^{2}}{4 n}\right\} \mathbf{P}\left\{\frac{\eta(N, n-N)}{n} \leqslant z\right\}+\delta,
$$

где величина $\delta$ может быть сделана сколь угодно малой выбором достаточно больших $n$, $A$ и достаточно малого $\varepsilon$.

Положим $y=b \sqrt{R} N / \sqrt{2 n}$. Из (28) и теоремы 1 следует, что

$$
\mathbf{P}\{\eta(G) / n \leqslant z\}=\sum_{\varepsilon \leqslant N / \sqrt{n} \leqslant A} \frac{b}{y^{2}} \sqrt{\frac{R}{2 n}} \sum_{s=0}^{\infty} \frac{(-1)^{s}}{s !} I_{s}\left(\frac{z}{y^{2}}, \frac{1}{y^{2}}\right)+\delta_{1},
$$

где величина $\delta_{1}$ может быть сделана сколь угодно малой выбором $n, A, \varepsilon$. Стоящее в правой части равенства (29) выражение представляет собой интегральную сумму функции

$$
y^{-2} \sum_{s=0}^{\infty}(-1)^{s}(s !)^{-1} I_{s}\left(z y^{-2}, y^{-2}\right)
$$

с шагом $b \sqrt{R /(2 n)}$. Поэтому из (29) получаем, что

$$
\begin{aligned}
\mathbf{P}\{\eta(G) / n \leqslant z\} & \rightarrow \int_{0}^{\infty} y^{-2} \sum_{s=0}^{\infty}(-1)^{s}(s !)^{-1} \\
& \times \int_{X_{s}\left(z y^{-2}, y^{-2}\right)} \frac{\exp \left\{-1 /\left(2\left(y^{-2}-x_{1}-\ldots-x_{s}\right)\right)\right\}}{(2 \pi)^{s / 2}\left(x_{1} \ldots x_{s}\left(y^{-2}-x_{1}-\ldots-x_{s}\right)\right)^{3 / 2}} d x_{1} \ldots d x_{s} d y .
\end{aligned}
$$

Интегрируя по у последнее выражение, получаем утверждение теоремы 4.

Рассмотрим теперь предельное поведение случайных величин $\mu_{r}(G)$. Нетрудно видеть, что

$$
\mathbf{P}\left\{\mu_{r}(G)=k\right\}=\sum_{N=k}^{n-k r} \mathbf{P}\{\lambda=N\} \mathbf{P}\left\{\mu_{r}(N, n-N)=k\right\}
$$


Отсюда и из леммы 3 следует, что

$$
\mathbf{P}\left\{\mu_{r}(G)=k\right\}=\sum_{\varepsilon \leqslant N / \sqrt{n} \leqslant A} \mathbf{P}\{\lambda=N\} \mathbf{P}\left\{\mu_{r}(N, n-N)=k\right\}+\delta,
$$

где величина $\delta$ может быть сделана сколь угодно малой выбором достаточно больших $n, A$ и достаточно малого $\varepsilon$. Из теоремы 3 , (7) и (8) следует, что при $n, r \rightarrow \infty$ так, что $r^{3} / n \rightarrow \infty$, равномерно по $N, \varepsilon \sqrt{n} \leqslant N \leqslant A \sqrt{n}$, выполняется соотношение $\mathbf{P}\left\{\mu_{r}(N, n-N)=0\right\} \rightarrow 1$, поэтому из (31) и леммы 3 следует утверждение теоремы 7 .

Пусть выполнены условия теоремы 5 . Из (30) получаем, что

$$
\left(p_{r} \sqrt{2 n} /(b \sqrt{R})\right) \mathbf{P}\left\{\mu_{r}(G)=k\right\}=S_{1}+S_{2}+S_{3},
$$

где

$$
\begin{aligned}
S_{i} & =\sum_{N \in M_{i}}\left(p_{r} \sqrt{2 n} /(b \sqrt{R})\right) \mathbf{P}\{\lambda=N\} \mathbf{P}\left\{\mu_{r}(N, n-N)=k\right\}, \quad i=1,2,3, \\
M_{1} & =\left\{N: k \leqslant N<z \sqrt{2 n} /(b \sqrt{R})-n^{7 / 24} p_{r}^{-5 / 12}\right\}, \\
M_{2} & =\left\{N: z \sqrt{2 n} /(b \sqrt{R})-n^{7 / 24} p_{r}^{-5 / 12} \leqslant N<z \sqrt{2 n} /(b \sqrt{R})+n^{7 / 24} p_{r}^{-5 / 12}\right\}, \\
M_{3} & =\left\{N: z \sqrt{2 n} /(b \sqrt{R})+n^{7 / 24} p_{r}^{-5 / 12} \leqslant N \leqslant n-k r\right\} .
\end{aligned}
$$

Мы увидим ниже, что основной вклад в сумму (32) вносит слагаемое $S_{2}$. Из леммы 3 следует, что

$$
S_{2}=p_{r} z e^{-z^{2} / 2}(1+o(1)) \sum_{N \in M_{2}} \mathbf{P}\left\{\mu_{r}(N, n-N)=k\right\}
$$

Полагая

$$
N=z \sqrt{2 n} /(b \sqrt{R})+y(2 n / R)^{1 / 4} \sqrt{\left(1-p_{r}\right) /\left(b p_{r}\right)},
$$

где

$$
|y| \leqslant\left(p_{r} \sqrt{n}\right)^{1 / 12}(R / 2)^{1 / 4} \sqrt{b /\left(1-p_{r}\right)},
$$

и используя теорему 2 , из (33) получаем, что

$$
S_{2}=\frac{z e^{-z^{2} / 2}(1+o(1))}{\sqrt{2 \pi z}} \sum_{N \in M_{2}}\left(\frac{R}{2 n}\right)^{1 / 4} \sqrt{\frac{b p_{r}}{1-p_{r}}} \exp \left\{-\frac{y^{2}}{2 z}\right\} .
$$

Нетрудно видеть, что сумма, стоящая в правой части (34), является интегральной для функции $\exp \left\{-y^{2} / 2 z\right\}$ с шагом $(R /(2 n))^{1 / 4}\left(b p_{r} /\left(1-p_{r}\right)\right)^{1 / 2}$. Отсюда следует, что

$$
S_{2} \rightarrow \sqrt{z /(2 \pi)} \int_{-\infty}^{\infty} \exp \left\{-\left(z^{2}+y^{2} / z\right) / 2\right\} d y=z e^{-z^{2} / 2} .
$$

Для завершения доказательства теоремы 5 нам осталось показать, что $S_{1}, S_{3} \rightarrow 0$. Из леммы 1 и (27) следует, что при $N \in M_{1}$

$$
\mathbf{P}\left\{\mu_{r}(N, n-N)=k\right\} \leqslant C_{1}\left(\begin{array}{l}
N \\
k
\end{array}\right) p_{r}^{k}\left(1-p_{r}\right)^{N-k} .
$$


Используя лемму 3 , находим, что $\mathbf{P}\{\lambda=N\} \leqslant C_{2} / \sqrt{n}$, поэтому из (35) получаем, что

$$
S_{1} \leqslant C_{3} \sum_{N \in M_{1}}\left(\begin{array}{l}
N \\
k
\end{array}\right) p_{r}^{k}\left(1-p_{r}\right)^{N-k}
$$

Из свойств биномиальных вероятностей следует, что слагаемые суммы (36) возрастают с ростом $N$, поэтому, опять используя теорему 2 и то, что число слагаемых в $S_{1}$ меньше, чем $z \sqrt{2 n} /(b \sqrt{R})$, находим, что

$$
S_{1} \leqslant C_{4} n^{1 / 4} \exp \left\{-y_{1}^{2} / 2\right\}
$$

где $y_{1}=\left(p_{r} \sqrt{n}\right)^{1 / 12}(R / 2)^{1 / 4} \sqrt{b /\left(1-p_{r}\right)}$. Отсюда следует, что $S_{1} \rightarrow 0$.

Рассмотрим $S_{3}$. Из (7) и (8) находим, что $\mathbf{E} \xi_{1}^{1 / 3}<\infty$. В этом случае, как показано в [11], справедлива оценка

$$
\max _{k} \mathbf{P}\left\{\zeta_{N}=k\right\} \geqslant C_{5} / N^{3}
$$

Отсюда и из леммы 1 получаем, что при $N \in M_{3}$

$$
\mathbf{P}\left\{\mu_{r}(N, n-N)=k\right\} \leqslant C_{6} n^{3}\left(\begin{array}{l}
N \\
k
\end{array}\right) p_{r}^{k}\left(1-p_{r}\right)^{N-k}
$$

Из этого неравенства, теоремы 2 и свойств биномиальных вероятностей находим, как и при оценке $S_{1}$, что

$$
S_{3} \leqslant C_{7} n^{7 / 2} \exp \left\{-y_{1}^{2} / 2\right\} \rightarrow 0
$$

Нам осталось доказать теорему 6. В силу (31) и леммы 3,

$$
\mathbf{P}\left\{\mu_{r}(G)=k t\right\}=\sum_{\varepsilon \leqslant N / \sqrt{n} \leqslant A} \frac{b^{2} R}{2} \frac{N}{n} \exp \left\{-\frac{b^{2} R N^{2}}{4 n}\right\} \mathbf{P}\left\{\mu_{r}(N, N-n)=k\right\}+\delta,
$$

где величина $\delta=\delta(n, A, \varepsilon)$ может быть сделана сколь угодно малой. Из теоремы 3 , (7) и (8) следует, что при $\varepsilon \sqrt{n} \leqslant N \leqslant A \sqrt{n}$ и $y=N b \sqrt{R /(2 n)}$

$$
\mathbf{P}\left\{\mu_{r}(N, N-n)=k\right\}=(k !)^{-1}(\gamma y)^{k} e^{\gamma y}(1+o(1))
$$

Отсюда и из (37) получаем, что

$$
\mathbf{P}\left\{\mu_{r}(G)=k\right\}=\sum_{\varepsilon \leqslant N / \sqrt{n} \leqslant A} \frac{\gamma^{k} y^{k+1}}{k !} \frac{b \sqrt{R}}{\sqrt{2 n}} \exp \left\{-\gamma y-\frac{y^{2}}{2}\right\}+o(1) .
$$

Поскольку в правой части последнего равенства стоит интегральная сумма функции $(\gamma y)^{k}\left(k ! e^{\gamma y}\right)^{-1} y e^{-y^{2} / 2}$ с шагом $b \sqrt{R /(2 n)}$, получаем утверждение теоремы 6.

Автор благодарен В. Ф. Колчину за постоянную поддержку. 


\section{Список литературы}

1. Колчин В. Ф., Случайные отображелия. Наука, Москва, 1984.

2. Степанов В. Е., Предельные распределения некоторых характеристик случайных отображений. Теория вероятностей и ее применения (1969) 14, №4, 639-653.

3. Павлов Ю. Л., Асимптотическое распределение максимального объема дерева в случайном лесе. Теория вероятностей и ее применения (1977) 22, №3, 523-533.

4. Павлов Ю. Л., Предельные теоремы для числа деревьев заданного объема в случайном лесе. Матем. сб. (1977) 103, №3, 392-403.

5. Harris B., Probability distributions related to random mappings. Ann. Math. Stat. (1960) 31, №4, 1045-1062.

6. Meir A., Moon J. W., On random mapping patterns. Combinatorica (1984) 4, №1, 61-70.

7. Харари Ф., Палмер Э., Перечисление графов. Мир, Москва, 1977.

8. Оттер Р., Число деревьев. В кн.: Перечислительные задачи комбинаторного анализа. Мир, Москва, 1979, с. 139-159.

9. Mutafchiev L. R., Limit theorem concerning random mapping patterns. Combinatorica (1988) 8, №4, 345-356.

10. Ибрагимов И. А., Линник Ю. В., Независимые и стачионарно связанные величины. Наука, Москва, 1965.

11. Essen C. G., On the concentration function of a sum of independent random variables. Z. Wahrscheinlichkeitstheor. Verw. Geb. (1968) 9, №4, 290-308.

Статья поступила 20.02.2004. 